\title{
Medida da distância reflexo margem por meio de processamento computadorizado de imagens em usuários de lentes de contato rígidas
}

\author{
Margin reflex distance measure by computerized image processing \\ in rigid contact lens wearers
}

\author{
Tiana Gabriela Burmann ${ }^{1}$ \\ Fabiana Borba Valiatti² \\ Zélia Maria Correa ${ }^{3}$ \\ Márcia Bayer ${ }^{4}$ \\ Ítalo Marcon ${ }^{5}$
}

\begin{tabular}{l} 
RESUMO \\
\hline Objetivo: Apresentar um método novo, baseado no processamento com- \\
putadorizado de imagens, para quantificar a distância reflexo margem \\
(MRD). Métodos: Selecionamos para o estudo pacientes do Setor de \\
Lentes de Contato do Serviço de Oftalmologia da Santa Casa de Porto \\
Alegre que foram divididos em dois grupos: o primeiro foi composto por \\
pacientes usuários de lentes de contato rígidas (63 olhos) e o segundo por \\
pacientes que foram encaminhados para adaptação de lentes de contato \\
sem história prévia de uso das mesmas (30 olhos). Todos os pacientes \\
foram fotografados com o auxílio de uma câmera fotográfica digital (Nikon \\
Coolpix 4300). A distância reflexo margem foi medida por processamento \\
computadorizado de imagens utilizando o programa Image J. Foram ex- \\
cluídos do estudo pacientes submetidos a cirurgias intra-oculares ou \\
palpebrais, pacientes apresentando ptose congênita e pacientes que ao \\
exame biomicroscópico apresentavam conjuntivite papilar gigante. Re- \\
sultados: O método utilizado para quantificar distância reflexo margem \\
parece bastante simples e aparentemente mais sensível e específico. O \\
valor médio da distância reflexo margem no grupo caso foi 2,46 mm e no \\
grupo controle 2,72 mm. Dessa forma, observou-se uma tendência de \\
diminuir a distância reflexo margem com o uso de lentes rígidas, embora \\
esses dados não tenham sido estatisticamente significativos (p=0,22). \\
Observa-se também que os valores de distância reflexo margem apresen- \\
tam uma variabilidade muito maior no grupo caso (41,46\%) do que no \\
controle (28,96\%), que se apresenta mais homogêneo. Conclusão: Esse \\
estudo introduziu uma metodologia inovadora para medir a distância \\
reflexo margem utilizando o processamento computadorizado de ima- \\
gens. Esse método é acessível e pode auxiliar no acompanhamento da \\
distância reflexo margem de pacientes usuários de lentes de contato, \\
especialmente rígidas.
\end{tabular}

Descritores: Lentes de contato; Blefaroptose; Processamento de imagem assistida por computador/métodos

\section{INTRODUÇÃ̃O}

Ptose consiste em uma patologia caracterizada pelo abaixamento da margem palpebral superior ${ }^{(1)}$. Diversos métodos já foram propostos com o objetivo de quantificar a ptose, incluindo a campimetria, a medida da distância entre a margem palpebral superior e o rebordo orbitário superior 
e a medida da fenda palpebral ${ }^{(1)}$. O limbo superior já foi considerado referencial para quantificar ptose, assim como a margem pupilar superior ${ }^{(2)}$. A distância da margem palpebral ao limbo também já foi utilizada para determinar a extensão da ressecção do músculo levantador na correção de ptoses congênitas ${ }^{(3)}$.

A medida da MRD (distância reflexo margem), que corresponde à distância (medida em milímetros com o uso de uma régua) entre a margem palpebral superior e o reflexo corneano formado por uma fonte luminosa puntiforme fixada na posição primária do olhar, tem sido utilizada para quantificar ptose.

A princípio, não existe um critério definitivo para se mensurar ptose. De acordo com alguns autores, valores de MRD inferiores a $2 \mathrm{~mm}$ ou assimetrias interpalpebrais iguais ou maiores a $2 \mathrm{~mm}$ não são normais ${ }^{(4)}$. A literatura sugere que o uso crônico de lentes de contato possa levar a um abaixamento da margem palpebral superior ${ }^{(5)}$.

O objetivo desse trabalho é apresentar um método novo e mais preciso para quantificar a MRD. Esse método, baseado no processamento computadorizado de imagens, foi testado em um grupo de pacientes usuários de lentes de contato rígidas e um grupo controle.

\section{MÉTODOS}

Foram examinados 34 pacientes do Setor de Lentes de Contato do Serviço de Oftalmologia da Santa Casa de Porto Alegre, totalizando 63 olhos de pacientes usuários de lentes de contato rígidas. $\mathrm{O}$ grupo controle foi composto por 15 indivíduos (30 olhos), sem história atual ou prévia de uso de lentes de contato, e que foram encaminhados para o Setor de Lentes de Contato para adaptação das mesmas. Foram excluídos do estudo pacientes com história de cirurgia intra-ocular prévia,conjuntivite papilar gigante, ptose congênita ou blefaroptose adquirida de causa traumática, miopática, anoftálmica e involucional. Todos os pacientes apresentavam exame biomicroscópico normal e assinaram o termo de consentimento livre e esclarecido.

Os pacientes foram fotografados com uma câmera digital Nikon Coolpix 4300. A MRD foi medida por meio de processamento computadorizado de imagens, utilizando o programa Image J. As imagens foram padronizadas por meio da colocação de uma etiqueta, de tamanho conhecido, na testa dos pacientes e controles (Figura 1).

\section{RESULTADOS}

O grupo caso incluiu 34 pacientes, sendo 20 do sexo feminino e 14 do masculino, com média de idade de 31 anos e o controle foi formado por 15 pacientes, sendo 10 femininos e 5 masculinos com média de idade de 29 anos.

A MRD foi medida por meio de processamento computadorizado de imagens. $\mathrm{O}$ valor médio da MRD no grupo caso foi 2,46 mm e no grupo controle 2,72 $\mathrm{mm}$. Dessa forma, observou-se uma tendência de diminuir a MRD com o uso de lentes rígidas, embora esses dados não tenham sido estatisticamente significativos $(\mathrm{p}=0,22)$. Observa-se também que os valores de MRD apresentam uma variabilidade muito maior no grupo caso $(41,46 \%)$ do que no controle $(28,96 \%)$, que se apresenta mais homogêneo.

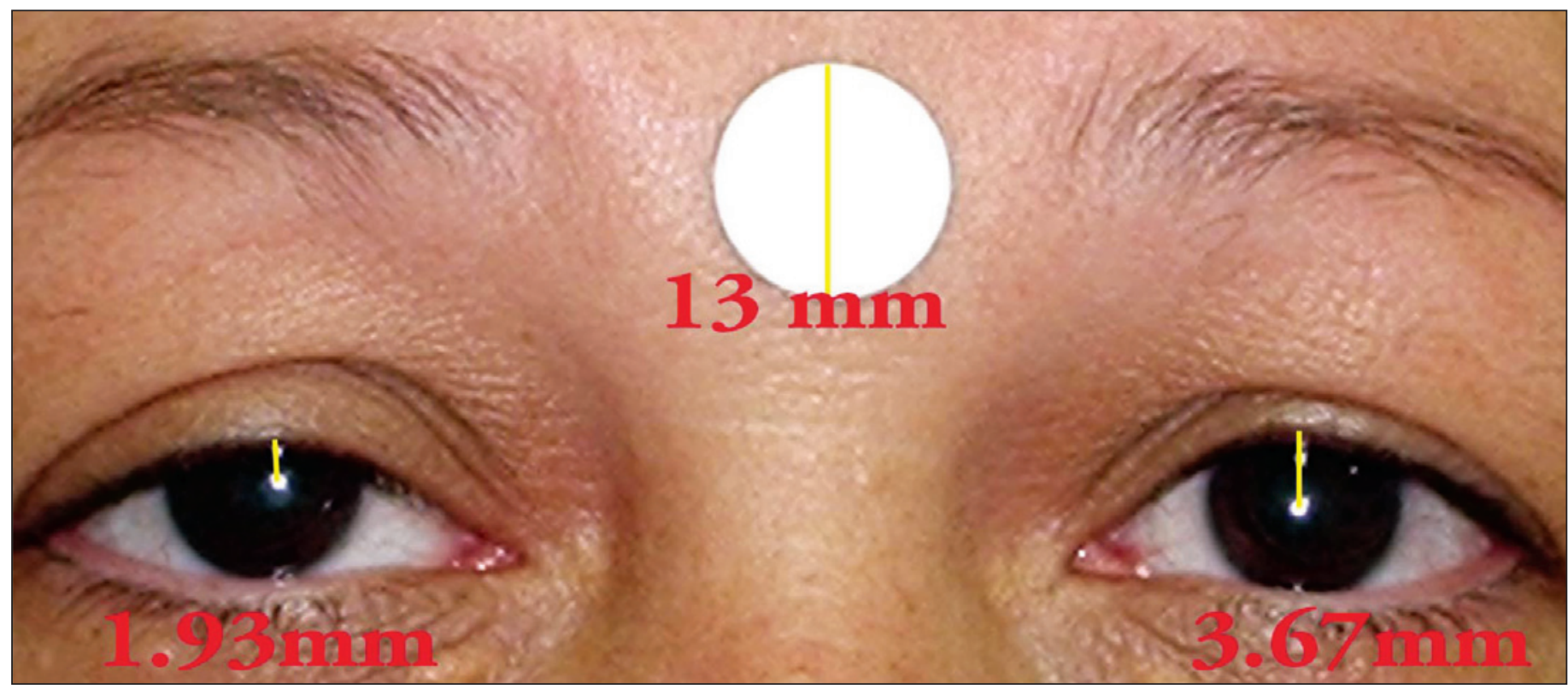

Figura 1 - Novo método utilizado para quantificar ptose, baseado na medida da MRD (distância do reflexo corneano formado por uma fonte de luz puntiforme e a margem palpebral superior) por meio de processamento computadorizado de imagens. A etiqueta na testa da paciente, de tamanho conhecido, serve para padronizar a imagem. 
Em relação ao grupo caso, a maioria (54 olhos) fazia uso diário das lentes de contato rígidas por várias horas (4 horas ou mais), 3 pacientes (5 olhos) faziam uso diário por poucas horas (3 horas ou menos) e dois pacientes (4 olhos) faziam uso ocasional das lentes. (Tabela 1). Uma vez que os grupos estudados eram pequenos, foi utilizado o teste de KruskalWallys (ANOVA não paramétrica) para análise estatística, que não mostrou correlação entre MRD e frequiência de uso das lentes de contato.

No grupo caso, a maioria (33 olhos) fazia uso das lentes por um período maior que 1 ano e igual ou menor a 5 anos, 6 pacientes ( 9 olhos) referiram fazer uso das LCR por um período igual ou menor a 1 ano, e o restante ( 21 olhos) fazia uso de lentes por um período superior a 5 anos e inferior a 10 anos, sendo que nenhum paciente do grupo fazia uso de lentes por mais de 10 anos. (Tabela 2). A análise estatística revelou que não existe associação entre o tempo de uso das lentes de contato (em meses) e a MRD (coeficiente de correlação de Pearson $\mathrm{p}=0,441)$.

O estudo revelou uma correlação direta entre tempo de uso da LCR e a freqüência diária de uso das lentes $(R=0,319$, correlação regular e direta), ou seja, pacientes usuários de lentes por vários meses são aqueles que utilizam as lentes várias horas.

Os pacientes usuários de lentes de contato rígidas foram separados em dois grupos, os que apresentavam ceratocone ( 38 olhos) e os sem ceratocone ( 25 olhos). De acordo com o teste t-Student para amostras independentes, podemos acreditar que não existe diferença estatística significativa entre as médias de MRD dos pacientes com e sem ceratocone $(\mathrm{p}=0,409)$.

\section{DISCUSS ÃO}

Os diversos métodos disponíveis para quantificar ptose sugerem que não exista um método padrão e universal. Atualmente, a medida da MRD, tem sido amplamente utilizada, no entanto, por acreditarmos que o uso da régua possa não ser um método muito preciso, sugerimos o uso de imagens nesse processo. A utilização do processamento computadorizado

\begin{tabular}{|ccc|}
\hline \multicolumn{4}{|c|}{ Tabela 1 - Horas de uso das lentes de contato rígidas nos 63 olhos } \\
estudados
\end{tabular}

\begin{tabular}{|ccc|}
\hline \multicolumn{3}{|c|}{ Tabela 2 - Anos de uso das lentes de contato rígidas nos 63 olhos } \\
estudados
\end{tabular}

de imagens já foi utilizado por outros autores para avaliar a fenda palpebral de pacientes com ptose congênita e para avaliar o contorno palpebral em pacientes com doença de Graves $^{(6-7)}$.

Esse método utilizado foi testado em pacientes usuários de lentes de contato rígidas por duas razões: a primeira porque a literatura sugere que esses pacientes possam apresentar abaixamento da margem palpebral superior e, eventualmente ptose, devido à desinserção do músculo levantador palpebral ${ }^{(5)}$ e a segunda porque em estudo prévio medimos a MRD com o método tradicional, utilizando uma régua.

O estudo mostrou uma tendência de redução da MRD com o uso de lentes de contato rígidas, embora não tenha sido comprovado estatisticamente, talvez porque a amostra estudada tenha sido pequena ou incluído poucos pacientes com história de uso de lente de contato rígida (LCR) por um tempo muito prolongado (acima de 10 anos nenhum paciente). Alguns estudos sugerem que o abaixamento da margem palpebral associado ao uso das LCR está relacionado ao longo tempo de uso das mesmas ${ }^{(5)}$.

A associação entre ptose e lentes de contato já foi mostrada por vários estudos inclusive prospectivos ${ }^{(8)}$. Alguns autores sugerem que a ptose nesses pacientes possa estar relacionada à manipulação excessiva e à dificuldade de colocar e retirar as lentes ${ }^{(8-9)}$.

Nesse estudo, os valores de MRD, tanto no grupo caso como no controle foram inferiores aos valores observados por outro estudo que utilizou a régua como método de medida da $\mathrm{MRD}^{(10)}$. Esse achado pode ser explicado pelas diferentes amostras analisadas, no entanto, ambos os estudos, demonstraram uma tendência de redução da MRD com o uso de lentes de contato rígidas.

A análise estatística não mostrou correlação de tempo (meses) e freqüência de uso (horas) com a medida da MRD, bem como não mostrou diferença estatisticamente significativa na medida da MRD de pacientes usuários de LCR com e sem ceratocone. Um estudo incluindo amostras maiores seria interessante para confirmar esses achados.

\section{CONCLUSÃO}

O estudo apresenta um método bastante efetivo, simples e de baixo custo para medir a MRD. Além de uma medida mais precisa, por meio desse método podemos acompanhar pacientes usuários de lentes de contato rígidas e, no caso de estarem diminuindo a MRD, suspender o seu uso ou orientar o paciente do risco de ptose. Esse método foi sugerido e testado em pacientes usuários de lentes de contato rígidas, mas pode ter aplicação no seguimento da MRD e da fenda palpebral de diversos pacientes. Outros estudos, com amostras maiores e mais homogêneas podem nos mostrar dados mais específicos em relação à associação entre ptose e uso de lentes de contato. 


\section{ABSTRACT}

Purpose: To measure the MRD (margin reflex distance) in rigid contact lens wearers and controls by a new method, based on computerized image processing. Method: The patients were selected from the Contact Lens Sector of the Ophthalmology Service at the "Complexo Hospitalar Santa Casa de Porto Alegre", and they were divided into two groups: the first was formed of rigid contact lens wearers (63 eyes) and the second of patients without previous history of contact lens wear (30 eyes). All patients were photographed with a digital camera (Nikon Coolpix 4300). The margin reflex distance was measured by a computerized image processing using the Image J program. The study excluded patients that underwent any kind of intraocular or eyelid surgery, patients with congenital ptosis and patients with giant papillae conjunctivitis. Results: The method utilized to measure margin reflex distance seems simple and more accurate. The average value of the margin reflex distance in the case group was $2.46 \mathrm{~mm}$ and in the control group $2.72 \mathrm{~mm}$. The study shows that there is a tendency of decreasing the margin reflex distance with contact lens wear although the data were not statistically significant $(\mathrm{p}=0.22)$. The margin reflex distance values show a greater variability in the case group $(41.46 \%)$ than in the control group $(28.96 \%)$, that is more homogeneous. Conclusion: This study introduced a new method to measure the margin reflex distance using computerized image processing. This method is accessible and could help in follow-up of the margin reflex distance in contact lens wearers, specially those rigid.

Keywords: Contact lenses; Blepharoptosis; Image processing computer-assisted/methods

\section{REFERÊNCIAS}

1. Cruz AA, Sobreira CF, Barreira AA. Blefaroptoses. In: Cruz AA, editor. Blefaroptoses e retrações palpebrais. Rio de Janeiro: Cultura Médica; c1997. p.36-101.

2. Beard C. Examination of the ptosis patient. In: Beard C., editor. Ptosis. St. Louis: Mosby; 1981. p.76-83.

3. Sarver BL, Putterman AM. Margin limbal distance to determine amount of levator resection. Arch Ophthalmol. 1985;103(3):354-6.

4. Small RG, Sabates NR, Burrows D. The measurement and definition of ptosis. Ophthal Plast Reconstr Surg. 1989;5(3):171-5.

5. van den Bosch WA, Lemij HG. Blepharoptosis induced by prolonged hard contact lens wear. Ophthalmology. 1992;99(12):1759-65.

6. Cruz AA, Lucchezi MC. Quantification of palpebral fissure shape in severe congenital blepharoptosis. Ophthal Plast Reconstr Surg. 1999;15(4):232-5.

7. Cruz AA, Coelho RP, Baccega A, Lucchezi MC, Souza AD, Ruiz EE. Digital image processing measurement of the upper eyelid contour in Graves disease and congenital blepharoptosis. Ophthalmology. 1998;105(5):913-8.

8. Júpiter D, Karesh J. Ptosis associated with PMMA/ rigid gás permeable contact lens wear. CLAO J. 1999;25(3):159-62.

9. Epstein G, Putterman AM. Acquired blepharoptosis secondary to contact-lens wear. Am J Ophthalmol. 1981;91(5):634-9.

10. Burmann TG, Valiatti FB, Obalski L, Marcon I. Blefaroptose em usuários de lentes de contato. Arq Bras Oftalmol. 2006;69(5):651-3.

\section{Simpósio Internacional de Oftalmologia da Santa Casa de São Paulo

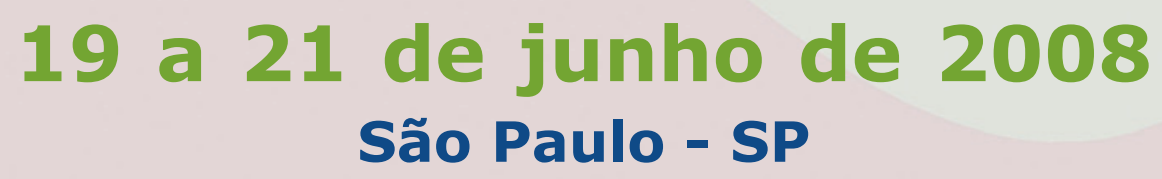

INFORMAÇÕES

JDE Comunicação e Eventos

Tels.: (11) 5084-9174/5082-3030 • Fax: (11) 5574-8261 E-mail: jdecomev@uol.com.br home page: www.oftalmosantasa.com.br 